\title{
VARIATIONAL INEQUALITIES FOR ENERGY FUNCTIONALS WITH NONSTANDARD GROWTH CONDITIONS
}

\author{
MARTIN FUCHS AND LI GONGBAO*
}

Abstract. We consider the obstacle problem

$$
\left\{\begin{array}{l}
\text { minimize } I(u)=\int_{\Omega} G(\nabla u) d x \text { among functions } u: \Omega \rightarrow R \\
\text { such that }\left.u\right|_{\partial \Omega}=0 \text { and } u \geq \Phi \text { a.e. }
\end{array}\right.
$$

for a given function $\Phi \in C^{2}(\bar{\Omega}),\left.\Phi\right|_{\partial \Omega}<0$ and a bounded Lipschitz domain $\Omega$ in $\mathbf{R}^{n}$. The growth properties of the convex integrand $G$ are described in terms of a $N$-function $A:[0, \infty) \rightarrow[0, \infty)$ with $\varlimsup_{t \rightarrow \infty} A(t) t^{-2}<\infty$. If $n \leq 3$, we prove, under certain assumptions on $G, C^{1, \alpha}$-partial regularity for the solution to the above obstacle problem. For the special case where $A(t)=t \ln (1+t)$ we obtain $C^{1, \alpha}$-partial regularity when $n \leq 4$. One of the main features of the paper is that we do not require any power growth of $G$.

\section{InTRODUCTION}

In this paper we discuss the obstacle problem

$$
\left\{\begin{array}{l}
\text { to minimize } I(u)=\int_{\Omega} G(\nabla u) d x \text { among functions } \\
u: \Omega \rightarrow R \text { s.t. }\left.u\right|_{\partial \Omega}=0 \text { and } u \geq \Phi \text { a.e. }
\end{array}\right.
$$

for a given function $\Phi \in C^{2}(\bar{\Omega})$ with the property $\left.\Phi\right|_{\partial \Omega}<0$, where $\Omega \subset \mathbb{R}^{n}$ is a bounded Lipschitz domain. The integrand $G: \mathbb{R}^{n} \rightarrow \mathbb{R}$ is assumed to be of class $C^{2}$ and locally coercive in the sense that

$$
D^{2} G(P)(Q, Q) \geq \lambda(P)|Q|^{2}, \quad \forall P, Q \in \mathbb{R}^{n},
$$

1991 Mathematics Subject Classification. 49N60, 35J70, 46E35.

Key words and phrases. Variational inequalities, nonstandard growth, Orlicz-Sobolev spaces, regularity theory.

* Partially supported by NSFC and Academy of Finland.

Received: September 15, 1997. 
holds with $\lambda(P)>0$. If the domain $\Omega \subset R^{n}$ is strictly convex, then the Hilbert-Haar theory applies showing that the unique minimizer $u$ is of class $C^{1, \alpha}(\Omega)$ for any $\alpha \in(0,1)$ (see $[\mathrm{KS}]$ ). For general $\Omega$ this result is only known to hold for integrands $G$ with power growth condition (see $\left[\mathrm{F}_{2}\right]$ ). The papers $[\mathrm{FS}]$ and $[\mathrm{FO}]$ investigated the regularity of local minimizers for vectorial problems without side conditions and integrands $G$ having nonstandard growth and proved (under certain additional assumptions on $G$ ) partial regularity in dimensions $n \geq 3$ and full regularity if $n=2$. These arguments do not immediately apply to problem (1.1) since then the Euler equation has to be replaced by a differential inequality or equivalently by a differential equation with a measure-valued r.h.s.. Using techniques outlined in $\left[\mathrm{F}_{1}\right]$ and $\left[\mathrm{F}_{2}\right]$ we first show that this measure has a well behaved density w.r.t. Lebesgue's measure so that we have a substitute for the Euler equation being valid in the unconstrained case. Unfortunately this step works only in the scalar case but nevertheless it can be combined with appropriate modifications of the quoted regularity arguments to give at least partial regularity of the minimizer up to a certain dimension $\mathrm{n}$ which can be calculated in terms of the integrand $G$.

Let us now give precise statements of the results: in what follows $\Omega \subset R^{n}$ will always denote a bounded Lipschitz domain and we also assume that the obstacle $\Phi$ is in the space $C^{2}(\bar{\Omega})$ satisfying $\left.\Phi\right|_{\partial \Omega}<0$.

To begin with, let us consider the logarithmic case

$$
G(Y):=|Y| \ln (1+|Y|), Y \in \mathbb{R}^{n} .
$$

Then problem (1.1) is well-posed on the class

$$
\mathbb{K}:=\{u \in \stackrel{\circ}{W} \underset{L L n L}{1}(\Omega): u \geq \Phi \text { a.e. }\},
$$

where $\stackrel{\circ}{W}_{L L n L}^{1}(\Omega)$ is the Orlicz-Sobolev space generated by the $N$-function $\mathrm{t} \ln (1+t)$ (compare Section 2 for definitions of Orlicz-Sobolev spaces), and we have

Theorem 1.1. Suppose that $G$ is given by (1.2).

a) Then problem (1.1) admits a unique solution $u \in \mathbb{K}$.

b) Suppose $n \leq 4$. Then there is an open subset $\Omega_{0} \subset \Omega$ such that $\mid \Omega-$ $\Omega_{0} \mid=0$ and $u \in C^{1, \alpha}\left(\Omega_{0}\right)$ for any $0<\alpha<1$. Here $|\cdot|$ denotes Lebesgue's measure of the set $\Omega-\Omega_{0}$.

Next, let A denote a $N$-function having the $\Delta_{2}$-property (compare [A]) and consider the corresponding Orlicz-Sobolev space $W_{A}^{1}(\Omega)$. The class of admissible functions is now defined as

$$
\mathbb{K}=\left\{u \in \stackrel{\circ}{W}{ }_{A}^{1}(\Omega): u \geq \Phi \text { a.e. }\right\},
$$

where ${ }_{W}^{W}{ }_{A}^{1}(\Omega)$ is the closure of $C_{0}^{\infty}(\Omega)$ w.r.t. Orlicz-Sobolev norm in $W_{A}^{1}(\Omega)$ (see Section 2). Concerning the integrand $G$ we require the following conditions to be satisfied with positive constants $C_{1}, \cdots, C_{5}, \lambda$ and a non-negative 
number $\mu$ :

$$
\begin{gathered}
G \text { is of class } C^{2} ; C_{1}(A(|E|)-1) \leq G(E) \leq C_{2}(A(|E|)+1) ; \\
G(E) \leq C_{3}\left(|E|^{2}+1\right) ; \\
|E|^{2}\left|D^{2} G(E)\right| \leq C_{4}(G(E)+1) ; \\
A^{*}(|D G(E)|) \leq C_{5}(A(|E|)+1) ; \\
D^{2} G(X)(Y, Y) \geq \lambda(1+|X|)^{-\mu}|Y|^{2}
\end{gathered}
$$

where $X, Y, E \in \mathbb{R}^{n}$ are arbitrary and $A^{*}$ denotes the $N$-function conjugate to $\mathrm{A}$ (see $[\mathrm{A}])$.

Theorem 1.2. Let (1.3)-(1.7) hold.

a) Then problem (1.1) possesses a unique solution $u \in \mathbb{K}$.

b) Suppose that $n \geq 2$ together with $\mu<\frac{4}{n}$. Then partial regularity in the sense of Theorem 1.1 b) is true.

The reader may wonder for what reason we state Theorem 1.1 since it seems to be a special case of Theorem 1.2 by letting $A(t):=t \ln (1+t), t \geq$ $0, G(Y):=A(|Y|)$. It is easily checked that (1.3)-(1.7) hold with $\mu=1$ so that by Theorem 1.2 we have partial regularity up to dimension 3. But Theorem 1.1 provides a slightly stronger result: partial regularity is also true in the 4-dimensional case which means that for the concrete model given by (1.2) direct calculations yield better results than the general theory summarized in Theorem 1.2.

Let us give some further examples of integrands $G$ satisfying (1.3)-(1.7):

Example 1. $A(t)=t^{p} \ln (1+t), t \geq 0,1 \leq p<2$;

$$
G(X)=\left\{\begin{array}{l}
A(|X|),|X| \geq 1 \\
g(|X|),|X| \leq 1
\end{array}, X \in \mathbb{R}^{n},\right.
$$

where $g(t)$ is the unique quadratic polynomial such that $G$ is $C^{2}$. In this case (1.7) holds for $\mu=2-p$

Example 2. $A(t)=t \ln (1+\ln (1+t)), t \geq 0 ; G(X):=A(|X|), X \in \mathbb{R}^{n}$.

Now for $\mu$ in (1.7) we may choose $1+\varepsilon$ with any number $\varepsilon>0$.

Example 3. $A(t)=\int_{0}^{t} s^{1-\alpha}(\arcsin h s)^{\alpha} d s, 0<\alpha \leq 1, t \geq 0$;

$$
G(X)=A(|X|), X \in \mathbb{R}^{n} .
$$

This model occurs in the study of certain generalized Newtonian fluids (see [BAH]), (1.7) now holds with $\mu=\alpha$. 
In all cases (1.6) may be verified as follows: recall the equation

$$
A^{*}\left(A^{\prime}(s)\right)=s A^{\prime}(s)-A(s)
$$

and observe $s A^{\prime}(s) \leq A(s)$ for large $s$ together with $A^{\prime}(|Q|)=|D G(Q)|$ for $|Q| \geq 1$.

Our paper is organized as follows: we only present a proof of Theorem 1.1 since the case of general integrands $G$ requires some minor modifications which can be found in [FO]. In Section 2 we introduce a quadratic regularization of problem (1.1) whose solutions converge to the minimizer of the problem under discussion. Section 3 describes the method of linearization which transforms the variational inequality for the obstacle problem into a nonlinear equation. In Section 4 we use this information to derive a Caccioppoli-type inequality which is the main tool for the regularity proof carried out in Section 5.

We finally wish to remark that our results can be viewed as a first step towards the regularity theory of obstacle problems with integrands $G$ being not of power growth. The standard growth condition is replaced by (1.3) which means that we can control $G$ in terms of a $N$-function $A$. Of course it is of great importance to discuss if singular points actually occur and if the restriction on the dimension $n$ is really needed. This investigation will be carried out in a subsequent paper.

\section{Some Comments on Function Spaces and Discussion of the REgularity PROBLEM}

We first give the definition of Orlicz-Sobolev spaces and state some results which we will use later. For a technical account of the Orlicz-Sobolev spaces we refer the reader to the books $[\mathrm{A}]$ and $[\mathrm{KR}]$.

As in $[\mathrm{A}]$ we say that a function $A:[0, \infty) \rightarrow[0, \infty)$ is a $N$-function if it satisfies the following properties $\left(N_{1}\right)$ and $\left(N_{2}\right)$ :

$\left(\mathbf{N}_{1}\right) \quad A$ is continuous, strictly increasing and convex;

$\left(\mathbf{N}_{2}\right) \lim _{t \rightarrow 0^{+}} A(t) / t=0, \lim _{t \rightarrow \infty} A(t) / t=\infty$.

We say that a $N$-function $A(t)$ satisfies a $\Delta_{2}$-condition near infinity if

$\left(\mathbf{N}_{3}\right)$ there exist positive constants $k$ and $t_{0}$ such that

$$
A(2 t) \leq k A(t) \text { for all } t \geq t_{0} .
$$

It is easy to see that $\left(N_{3}\right)$ implies the inequality

$$
A(\lambda t) \leq A\left(\lambda t_{0}\right)+\left(1+k^{\frac{\ln \lambda}{\ln 2}+1}\right) A(t)
$$

being valid for all $t, \lambda \geq 0$.

Let $A(t)$ be a $N$-function. Then the conjugate $A^{*}$ of $A$ is defined as

$$
A^{*}(s)=\max _{t \geq 0}(s t-A(t)) .
$$

It is easy to see that $A^{*}$ is also a $N$-function.

For a bounded domain $\Omega$, the Orlicz space $L_{A}(\Omega)$ is defined as $L_{A}(\Omega):=\left\{u: \Omega \rightarrow R\right.$ measurable $\mid \exists \lambda>0$ such that $\left.\int_{\Omega} A(\lambda|u|) d x<+\infty\right\}$. 
$L_{A}(\Omega)$ together with the Luxemburg norm

$$
\|u\|_{L_{A}(\Omega)}=\inf \left\{l>0: \int_{\Omega} A\left(\frac{|u|}{l}\right) d x \leq 1\right\}
$$

carries the structure of a Banach space.

Let $E_{A}(\Omega)$ be the closure in $L_{A}(\Omega)$ of all bounded measurable functions. Then $E_{A}(\Omega)$ is a separable linear subspace of $L_{A}(\Omega)$, moreover, $L_{A}(\Omega)=$ $E_{A}(\Omega)$ iff $A$ satisfies a $\Delta_{2}$-condition near infinity (see $[\mathrm{A}]$ ).

The Orlicz-Sobolev space generated by a $N$-function $A$ is defined as

$$
W_{A}^{1}(\Omega)=\left\{u: \Omega \rightarrow R \text { measurable }|u,| \nabla u \mid \in L_{A}(\Omega)\right\}
$$

which together with the norm

$$
\|u\|_{W_{A}^{1}(\Omega)}=\|u\|_{L_{A}(\Omega)}+\||\nabla u|\|_{L_{A}(\Omega)}
$$

is a Banach space.

We further let

$$
\stackrel{\circ}{W}{ }_{A}^{1}(\Omega):=\text { closure of } C_{0}^{\infty}(\Omega) \text { in } W_{A}^{1}(\Omega) \text { w.r.t. }\|\cdot\|_{W_{A}^{1}(\Omega)} .
$$

The following results were proved in $[\mathrm{FO}]$.

Lemma 2.1. (Theorem 2.1 in [FO]) Let $\Omega$ be a bounded Lipschitz domain and suppose that $A(t)$ is a $N$-function satisfying a $\Delta_{2}$-condition near infinity. Then we have

$$
\stackrel{\circ}{W}{ }_{A}^{1}(\Omega)=W_{A}^{1}(\Omega) \cap \stackrel{\circ}{W}_{A}^{1}(\Omega) .
$$

Lemma 2.2. (Lemma 2.4 in [FO], Poincare's inequality) For $u \in \stackrel{\circ}{W}{ }_{A}^{1}(\Omega)$ we have the inequality

$$
\|u\|_{L_{A}(\Omega)} \leq d\||\nabla u|\|_{L_{A}(\Omega)}
$$

where $d$ is the diameter of $\Omega$.

It is easy to see that the following result is true (see $[\mathrm{A}]$ or $[\mathrm{KR}]$ ).

Lemma 2.3. Let $\Omega$ be a bounded domain in $\mathbb{R}^{n}$ and $A$ be a $N$-function satisfying a $\Delta_{2}$-condition near infinity. Consider a sequence $\left\{u_{m}\right\}$ in $L_{A}(\Omega)$. Then the following conditions are equivalent:

(a) $\int_{\Omega} A\left(\left|u_{m}\right|\right) d x \rightarrow 0$ as $m \rightarrow \infty$;

(b) $\int_{\Omega} A\left(\lambda\left|u_{m}\right|\right) d x \rightarrow 0$ as $m \rightarrow \infty$ for any $\lambda \geq 0$ and

(c) $\lim _{m \rightarrow \infty}\left\|u_{m}\right\|_{L_{A}(\Omega)}=0$.

Let $A$ be a $N$-function with conjugate function $A^{*}$. A sequence $\left\{u_{m}\right\}$ in

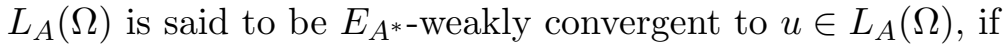

$$
\lim _{m \rightarrow \infty} \int_{\Omega} u_{m} v d x=\int_{\Omega} u v d x, \forall v \in E_{A^{*}}(\Omega) .
$$


A sequence $\left\{u_{m}\right\}$ in $W_{A}^{1}(\Omega)$ is said to be $E_{A^{*} \text {-weakly convergent to some }}$ $u \in W_{A}^{1}(\Omega)$ if both $u_{m}-u$ and $\nabla u_{m}-\nabla u$ are $E_{A^{*} \text {-weakly convergent to } 0}$ in $L_{A}(\Omega)$.

The following results can be found in $[\mathrm{KR}]$.

Lemma 2.4. Let $\Omega$ be a bounded domain in $R^{n}$ and $A$ be a $N$-function with conjugate function $A^{*}$. Then the following statements hold:

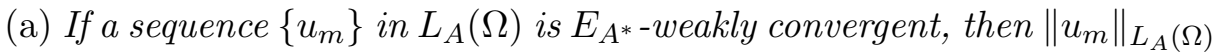
$\leq C$ for some constant $C$ and any $m \geq 1$;

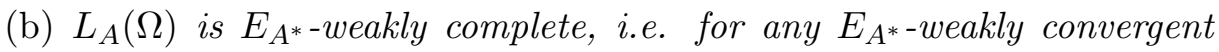
sequence $\left\{u_{m}\right\}$ in $L_{A}(\Omega)$, there is a unique $u \in L_{A}(\Omega)$ such that

$$
\lim _{m \rightarrow+\infty} \int_{\Omega} u_{m}(x) v(x) d x=\int_{\Omega} u(x) v(x) d x, \forall v \in E_{A^{*}}(\Omega)
$$

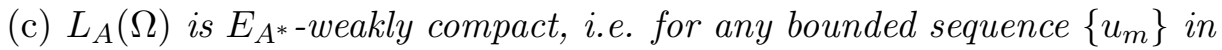

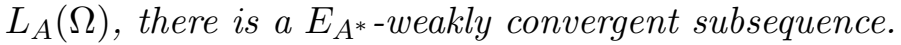

It is easy to prove the following results.

Lemma 2.5. Let $A$ denote a $N$-function. Then

(a) the following imbeddings

$$
\begin{gathered}
W_{A}^{1}(\Omega) \hookrightarrow W_{1}^{1}(\Omega), \\
\stackrel{\circ}{W}{ }_{A}^{1}(\Omega) \hookrightarrow \stackrel{\circ}{W}_{1}^{1}(\Omega)
\end{gathered}
$$

are continuous.

(b) If $\left\{u_{m}\right\}$ is a bounded sequence in $W_{A}^{1}(\Omega)\left(\stackrel{\circ}{W}{ }_{A}^{1}(\Omega)\right)$, then there is a $u \in W_{A}^{1}(\Omega)\left(\stackrel{\circ}{W}{ }_{A}^{1}(\Omega)\right)$ and a subsequence $\left\{u_{m}\right\}$ (still denoted by $\left\{u_{m}\right\}$ ) such that

$$
u_{m} \rightarrow u \quad E_{A^{*}}-\text { weakly in } W_{A}^{1}(\Omega)\left(\stackrel{\circ}{W}{ }_{A}^{1}(\Omega)\right)
$$

and

$$
u_{m} \rightarrow u \text { weakly in } W_{1}^{1}(\Omega)(\stackrel{\circ}{W} \underset{1}{1}(\Omega)) .
$$

Lemma 2.6. Let $\Omega \subset \mathbb{R}^{n}$ be a bounded Lipschitz domain and $A$ be a $N$ function satisfying a $\Delta_{2}$-condition near infinity.

(a) If $u, v \in W_{A}^{1}(\Omega)\left(\stackrel{\circ}{W}{ }_{A}^{1}(\Omega)\right)$, then both $\max (u, v)$ and $\min (u, v)$ are in $W_{A}^{1}(\Omega)\left(\stackrel{\circ}{W}{ }_{A}^{1}(\Omega)\right)$ with

$$
\nabla \max (u, v)= \begin{cases}\nabla u(x) & \text { if } u(x) \geq v(x) \\ \nabla v(x) & \text { if } v(x) \geq u(x)\end{cases}
$$

and

$$
\nabla \min (u, v)(x)=\left\{\begin{array}{ll}
\nabla u(x) & \text { if } u(x) \leq v(x) \\
\nabla v(x) & \text { if } v(x) \leq u(x)
\end{array} .\right.
$$


(b) If $u_{j}, v_{j} \in W_{A}^{1}(\Omega)\left(\stackrel{\circ}{W}{ }_{A}^{1}(\Omega)\right)$ with $u_{j} \rightarrow u, v_{j} \rightarrow v$ in $W_{A}^{1}(\Omega)\left(\stackrel{\circ}{W}{ }_{A}^{1}(\Omega)\right)$, then $\max \left(u_{j}, v_{j}\right) \rightarrow \max (u, v)$ and $\min \left(u_{j}, v_{j}\right) \rightarrow \min (u, v)$ in $W_{A}^{1}(\Omega)$ $\left(\stackrel{\circ}{W}{ }_{A}^{1}(\Omega)\right)$.

Proof. We mention only that since A satisfies a $\Delta_{2}$-condition, Lemma 2.3 can be used. The proof will then be carried out as in the ordinary Sobolev space case. (See e.g. [HKM]).

We now turn to our main problem (1.1):

$$
\left\{\begin{array}{l}
\text { to find } u \in \mathbb{K}=\left\{v \in \stackrel{\circ}{W}{ }_{A}^{1}(\Omega) \mid v \geq \Phi \text { a.e }\right\} \\
\text { such that } I(u)=\inf _{v \in \mathbb{K}} I(v) \text { where } I(w)=\int_{\Omega} G(\nabla w) \mathrm{dx} \text { and } G \\
\text { satisfies (1.3)-(1.7) for some } N \text { - function with }\left(N_{1}\right),\left(N_{2}\right),\left(N_{3}\right) .
\end{array}\right.
$$

The solvability of (1.1) is given by the following

Theorem 2.7. Problem (1.1) admits a unique solution $u$.

Proof. Since $\left.\Phi\right|_{\partial \Omega}<0$ and $\Phi \in C^{2}(\bar{\Omega}), v=\max (0, \Phi) \in \stackrel{\circ}{W} \stackrel{2}{2}(\Omega) \hookrightarrow \stackrel{\circ}{W}{ }_{A}^{1}(\Omega)$ with $v \geq \Phi$ a.e, so $v \in \mathbb{K}$ and $\mathbb{K} \neq \phi$.

Let $\left\{u_{m}\right\}$ be a minimizing sequence in $\mathbb{K}$ of $\mathrm{I}$, then

$$
I\left(u_{m}\right) \rightarrow \inf _{v \in \mathbb{K}} I=\gamma
$$

and $\int_{\Omega} G\left(\nabla u_{m}\right) d x \leq C$. By (1.3) we see that

$$
\int_{\Omega} A\left(\left|\nabla u_{m}\right|\right) d x \leq C<+\infty \quad \forall m .
$$

Since $A$ satisfies a $\Delta_{2}$-condition, we have $\left\|\left|\nabla u_{m}\right|\right\|_{L_{A}(\Omega)} \leq C$ (see $[\mathrm{A}]$ or $[\mathrm{KR}]$ ). The Poincare inequality (Lemma 2.2) implies that

$$
\left\|u_{m}\right\|_{W_{A}^{1}(\Omega)} \leq C .
$$

Using Lemma 2.5 we find a function $\hat{u} \in \stackrel{\circ}{W}{ }_{A}^{1}(\Omega)$ and a subsequence $\left\{u_{m}\right\}$ such that

$$
u_{m} \rightarrow u \quad \text { in } \quad \stackrel{\circ}{W} \stackrel{1}{1}(\Omega) .
$$

Sobolev's imbedding implies that

$$
u_{m} \rightarrow u \text { a.e. in } \Omega,
$$

hence $u \geq \Phi$ and $u \in \mathbb{K}$.

According to Mazur's Lemma we can arrange

$$
v_{m}=\sum_{j=m}^{N(m)} c_{j}^{m} u_{j} \rightarrow u \text { in } W_{1}^{1}(\Omega)
$$


for suitable sequences $N(m) \in \mathbf{N}, c_{j}^{m} \geq 0, \sum_{j=m}^{N(m)} c_{j}^{m}=1$, and for some subsequence we may also assume

$$
\nabla v_{m} \rightarrow \nabla u \quad \text { a.e. }
$$

The convexity of $G(X)$ implies that

$$
I(u) \leq \gamma,
$$

and the strict convexity of $G$ gives the uniqueness of the minimizer.

In what follows we let $G(E)=|E| \ln (1+|E|), A(t)=t \ln (1+t)$ for $t \geq 0$. To study the regularity problem we define

$$
G_{\delta}(E)=\frac{\delta}{2}|E|^{2}+G(E)
$$

for $E \in R^{n}, \delta>0$. We further let

$$
\begin{gathered}
\mathbb{K}^{*}=\{w \in \stackrel{\circ}{W} \underset{2}{1}(\Omega) \mid w \geq \Phi \text { a.e. in } \Omega\}, \\
I_{\delta}(w)=\int_{\Omega} G_{\delta}(\nabla w) d x .
\end{gathered}
$$

We have the following density result.

Lemma 2.8. $\mathbb{K}^{*}$ is dense in $\mathbb{K}$ w.r.t. the norm $\|\cdot\|_{W_{A}^{1}(\Omega)}$.

Proof. For any $u \in \mathbb{K}$, since $\Phi \in C^{2}(\bar{\Omega})$ and $\left.\Phi\right|_{\partial \Omega}<0$, we have $\max (0, \Phi) \in$ $\stackrel{\circ}{W} \underset{2}{1}(\Omega)$, hence

$$
u-\max (0, \Phi) \in \stackrel{\circ}{W}{ }_{A}^{1}(\Omega) .
$$

By the definition of $\stackrel{\circ}{W}_{A}^{1}(\Omega)$, there is a sequence $v_{i} \in C_{0}^{\infty}(\Omega)$ such that

$$
v_{i} \rightarrow u-\max (0, \Phi) \text { in } \stackrel{\circ}{W}{ }_{A}^{1}(\Omega) .
$$

Since $\Phi-\max (0, \Phi)<0$ in a neighborhood $N$ of $\partial \Omega$, we see

$$
\max \left(v_{i}, \Phi-\max (0, \Phi)\right)=0 \text { in }\left(\Omega \backslash \operatorname{spt}\left(v_{i}\right)\right) \cap N .
$$

In fact we have

$$
\max \left(v_{i}, \Phi-\max (0, \Phi)\right) \in \stackrel{\circ}{W}{ }_{2}^{1}(\Omega) \hookrightarrow \stackrel{\circ}{W}{ }_{A}^{1}(\Omega) .
$$

Let $u_{i}=\max \left(v_{i}, \Phi-\max (0, \Phi)\right)+\max (0, \Phi)$. Then $u_{i} \in \mathbb{K}^{*}$ and by Lemma 2.6

$$
\begin{aligned}
u_{i} & \rightarrow \max (u-\max (0, \Phi), \Phi-\max (0, \Phi))+\max (0, \Phi) \\
& =u-\max (0, \Phi)+\max (0, \Phi)=u \text { in } W_{A}^{1}(\Omega) .
\end{aligned}
$$

This proves the Lemma.

We have the following result concerning the functional $I_{\delta}(w)$. 
Theorem 2.9. a) The problem $I_{\delta} \rightarrow \min$ in $\mathbb{K}^{*}$ has a unique solution $u_{\delta}$. b) We have $u_{\delta} \rightarrow u$ in $W_{1}^{1}(\Omega)$, moreover, $I_{\delta}\left(u_{\delta}\right) \rightarrow I(u)$ as $\delta \downarrow 0$, and

$$
\frac{\delta}{2} \int_{\Omega}\left|\nabla u_{\delta}\right|^{2} d x \rightarrow 0
$$

where $u$ is the minimizer of $I(v)$ in $\mathbb{K}$.

Proof. Since $\mathbb{K}^{*} \neq \phi$, we may apply the direct method in order to verify part a).

Let $w \in \mathbb{K}^{*}$ be fixed. Then for $\delta<1$

$$
I_{\delta}\left(u_{\delta}\right) \leq I_{\delta}(w) \leq I_{1}(w)=C_{1}
$$

which implies

$$
I\left(u_{\delta}\right) \leq C
$$

and as in the proof of Theorem 2.7 we see

$$
u_{\delta} \rightarrow \tilde{u} \text { weakly in } \stackrel{\circ}{W} \underset{1}{1}(\Omega)
$$

for some $\tilde{u} \in \stackrel{\circ}{W}{ }_{A}^{1}(\Omega)$ which belongs to the class $\mathbb{K}$.

Then, for any $w \in \mathbb{K}^{*}$, we have

$$
I_{\delta}\left(u_{\delta}\right) \leq I_{\delta}(w) \underset{\delta \rightarrow 0^{+}}{\longrightarrow} I(w)
$$

and

$$
\begin{aligned}
I(\tilde{u}) & \leq \lim _{\delta \rightarrow 0^{+}} I\left(u_{\delta}\right) \leq \lim _{\delta \rightarrow 0^{+}} I_{\delta}\left(u_{\delta}\right)=: \alpha \\
& \leq \overline{\lim }_{\delta \rightarrow 0^{+}} I_{\delta}\left(u_{\delta}\right)=: \beta,
\end{aligned}
$$

so that

$$
I(\tilde{u}) \leq \alpha \leq \beta \leq I(w), \forall w \in \mathbb{K}^{*} .
$$

By Lemma $2.8, \mathbb{K}^{*}$ is dense in $\mathbb{K}$, thus we have $\tilde{u}=u$.

Remark 2.10. We mention that the proof of Lemma 2.9 also applies to general integrands $G$ with (1.3)-(1.7) and $A$ satisfying a $\Delta_{2}$-condition near infinity.

We now state a higher integrability result.

Theorem 2.11. For the minimizer u from Theorem 2.7 we have

$$
\sqrt{1+|\nabla u|} \in W_{2, \mathrm{loc}}^{1}(\Omega)
$$

Corollary 2.12. $\nabla u$ is in the space $L_{\mathrm{loc}}^{p}\left(\Omega, \mathbb{R}^{n}\right)$ for

$$
p\left\{\begin{array}{lll}
<\infty & \text { if } & n=2 \\
\leq \frac{n}{n-2} & \text { if } & n \geq 3
\end{array}\right.
$$


Corollary 2.13. If $n=2$, then $u \in C^{0, \alpha}(\Omega)$ for any $0<\alpha<1$; if $n \leq 4$, then $\nabla u \in L_{\text {loc }}^{2}(\Omega)$.

Remark 2.14. In the general case we have instead of Theorem 2.11 that $(1+|\nabla u|)^{1-\mu / 2} \in W_{2, \text { loc }}^{1}(\Omega)$ (compare [FO] for the unconstrained case).

Proof of Theorem 2.11. We fix a coordinate direction $e_{\gamma} \in \mathbb{R}^{n}, \gamma=1, \cdots, n$, and define for $h \neq 0$ and functions $f$

$$
\Delta_{h} f(x)=\frac{1}{h}\left(f\left(x+h e_{\gamma}\right)-f(x)\right) .
$$

Let $\left\{u_{\delta}\right\}$ denote the sequence obtained in Theorem 2.9. With $\delta$ fixed we consider $\varepsilon>0$ satisfying $\varepsilon h^{-2}<\frac{1}{2}$ and define

$$
v_{\varepsilon}:=u_{\delta}+\varepsilon \Delta_{-h}\left(\eta^{2} \Delta_{h}\left[u_{\delta}-\Phi\right]\right)
$$

with $\eta \in C_{0}^{2}(\Omega)$ such that $0 \leq \eta \leq 1$. Then $v_{\varepsilon} \in \mathbb{K}^{*}$, hence $I_{\delta}\left(u_{\delta}\right) \leq I_{\delta}\left(v_{\varepsilon}\right)$, and we deduce

$$
\int_{\Omega} \frac{1}{\varepsilon}\left[G_{\delta}\left(\nabla u_{\delta}+\varepsilon \nabla\left(\Delta_{-h}\left(\eta^{2} \Delta_{h}\left(u_{\delta}-\Phi\right)\right)\right)\right)-G_{\delta}\left(\nabla u_{\delta}\right)\right] d x \geq 0 .
$$

Letting $\varepsilon \rightarrow 0$ we infer

$$
\int_{\Omega} D G_{\delta}\left(\nabla u_{\delta}\right) \cdot \nabla\left(\Delta_{-h}\left[\eta^{2} \Delta_{h}\left[u_{\delta}-\Phi\right]\right]\right) d x \geq 0
$$

where $D G_{\delta}$ denotes the gradient of $G_{\delta}$. Using "integration by parts" for $\Delta_{-h}$ we end up with the result

$$
\int_{\Omega} \Delta_{h}\left\{D G_{\delta}\left(\nabla u_{\delta}\right)\right\} \cdot \nabla\left(\eta^{2} \Delta_{h}\left[u_{\delta}-\Phi\right]\right) d x \leq 0 .
$$

Introducing $\xi_{t}=\nabla u_{\delta}+t h \Delta_{h}\left(\nabla u_{\delta}\right)$ we may write

$$
\begin{aligned}
& \Delta_{h}\left\{D G_{\delta}\left(\nabla u_{\delta}\right)\right\} \cdot \nabla\left(\eta^{2} \Delta_{h}\left[u_{\delta}-\Phi\right]\right) \\
= & \int_{0}^{1} D^{2} G_{\delta}\left(\xi_{t}\right)\left(\Delta_{h} \nabla u_{\delta}, \nabla\left(\eta^{2} \Delta_{h}\left[u_{\delta}-\Phi\right]\right) d t .\right.
\end{aligned}
$$

Let us further define the bilinear form

$$
B_{x}(X, Y)=\int_{0}^{1} D^{2} G_{\delta}\left(\xi_{t}(x)\right)(Z, Y) d t
$$

for $x \in \Omega$ and $X, Y \in \mathbb{R}^{n}$. Then (2.15) takes the form

$$
\int_{\Omega} B_{x}\left(\Delta_{h} \nabla u_{\delta}, \nabla\left(\eta^{2} \Delta_{h}\left[u_{\delta}-\Phi\right]\right) d x \leq 0 .\right.
$$

We have

$$
\nabla\left(\eta^{2} \Delta_{h} u_{\delta}\right)=\eta^{2} \nabla \Delta_{h} u_{\delta}+2 \eta \nabla \eta \Delta_{h} u_{\delta}
$$

and (2.16) implies

$$
\begin{aligned}
& \int_{\Omega} \eta^{2} B_{x}\left(\Delta_{h} \nabla u_{\delta}, \Delta_{h} \nabla u_{\delta}\right) d x \\
\leq & \int_{\Omega} B_{x}\left(\Delta_{h} \nabla u_{\delta}, \nabla\left(\eta^{2} \Delta_{h} \Phi\right)-2 \eta \nabla \eta \Delta_{h} u_{\delta}\right) d x .
\end{aligned}
$$


Using the Cauchy-Schwarz's inequality in the form

$$
B_{x}(X, Y) \leq B_{x}(X, X)^{\frac{1}{2}} B_{x}(Y, Y)^{\frac{1}{2}}
$$

together with Young's inequality we arrive at

$$
\begin{aligned}
& \int_{\Omega} \eta^{2} B_{x}\left(\Delta_{h} \nabla u_{\delta}, \Delta_{h} \nabla u_{\delta}\right) d x \\
\leq & C_{1}(\eta) \int_{\text {spt } \eta}\left\|B_{x}\right\|\left(\left|\Delta_{h} \Phi\right|^{2}+\left|\nabla \Delta_{h} \Phi\right|^{2}+\left|\Delta_{h} u_{\delta}\right|^{2}\right) d x
\end{aligned}
$$

for some constant $C_{1}$ depending also on $\eta$.

It is easy to check (see $[\mathrm{FS}]$ ) that the following bounds hold for the parameter dependent bilinear form $D^{2} G_{\delta}(Z)(X, Y)$

$$
\begin{aligned}
& \left\|D^{2} G_{\delta}(Z)\right\|=\sup _{\|X\|=1} D^{2} G_{\delta}(Z)(X, X) \leq \delta+\frac{2 \ln (1+|Z|)}{|Z|} \\
& D^{2} G_{\delta}(Z)(X, X) \geq \delta|X|^{2}+(1+|Z|)^{-1}|X|^{2} \geq \delta|X|^{2} .
\end{aligned}
$$

Inserting this into (2.17), we find that

$$
\int_{\Omega} \eta^{2}\left|\Delta_{h} \nabla u_{\delta}\right|^{2} d x \leq C_{3}(\delta, \eta)\left\{\left\|u_{\delta}\right\|_{W_{2}^{1}(\Omega)}^{2}+\|\Phi\|_{W_{2}^{2}(\Omega)}^{2}\right\}
$$

and therefore $u_{\delta} \in W_{2, \operatorname{loc}(\Omega)}^{2}$. For this reason we can replace $\Delta_{h}$ in $(2.16)$ by the partial derivative $\partial_{\gamma}$. Then, following the calculation after $(2.16)$, we see that $(2.17),(2.20)$ have to be replaced by (summation over $\gamma$ )

$$
\begin{aligned}
& \int_{\Omega} \eta^{2} D^{2} G_{\delta}\left(\nabla u_{\delta}\right)\left(\partial_{\gamma} \nabla u_{\delta}, \partial_{\gamma} \nabla u_{\delta}\right) d x \\
\leq & C_{4}(\eta) \int_{\text {spt }}|| D^{2} G_{\delta}\left(\nabla u_{\delta}\right) \|\left(\left.|| \nabla \Phi\right|^{2}+\left|\nabla^{2} \Phi\right|^{2}+\left|\nabla u_{\delta}\right|^{2}\right) d x \\
\leq & C_{5}(\eta)\left[\delta \int_{\Omega}\left(\left|\nabla u_{\delta}\right|^{2}+|\nabla \Phi|^{2}+\left|\nabla^{2} \Phi\right|^{2}\right) d x\right. \\
& \left.+C_{6}(\eta)\left(\int_{\Omega}\left|\nabla u_{\delta}\right| \ln \left(1+\left|\nabla u_{\delta}\right|\right) d x+\|\Phi\|_{W_{2}^{2}(\Omega)}^{2}\right)\right]
\end{aligned}
$$

where $C_{6}$ is independent of $\delta$. We know that $\delta \int_{\Omega}\left|\nabla u_{\delta}\right|^{2} d x \rightarrow 0$ as $\delta \rightarrow 0+$ (cf. Theorem 2.9) and $\sup _{\delta>0} \int_{\Omega}\left|\nabla u_{\delta}\right| \ln \left(1+\left|\nabla u_{\delta}\right|\right) d x<\infty$. Hence (2.21) implies

$$
\int_{\Omega^{\prime}} D^{2} G_{\delta}\left(\nabla u_{\delta}\right)\left(\partial_{\gamma} \nabla u_{\delta}, \partial_{\gamma} \nabla u_{\delta}\right) d x \leq C\left(\Omega^{\prime}\right)
$$

for any subdomain $\Omega^{\prime} \subset \subset \Omega$.

Combining (2.19) and (2.22) we find that

$$
\int_{\Omega^{\prime}}\left|\nabla \sqrt{1+\left|\nabla u_{\delta}\right|}\right|^{2} d x \leq C\left(\Omega^{\prime}\right) .
$$

Thus there is a function $\omega \in W_{2 \text {,loc }}^{1}(\Omega)$ such that

$$
\sqrt{1+\left|\nabla u_{\delta}\right|} \rightarrow \omega \text { in } W_{2, \text { loc }}^{1}(\Omega) .
$$


We claim

$$
\omega=\sqrt{1+|\nabla u|}
$$

To show $(2.24)$, we note that

$$
\begin{aligned}
& I_{\delta}\left(u_{\delta}\right)-I(u)=\frac{\delta}{2} \int_{\Omega}\left|\nabla u_{\delta}\right|^{2} d x+I\left(u_{\delta}\right)-I(u) \\
& =\frac{\delta}{2} \int_{\Omega}\left|\nabla u_{\delta}\right|^{2} d x+\int_{\Omega} D G(\nabla u)\left(\nabla u_{\delta}-\nabla u\right) d x \\
& \quad+\int_{\Omega} \int_{0}^{1} D^{2} G\left((1-t) \nabla u+t \nabla u_{\delta}\right)\left(\nabla u_{\delta}-\nabla u, \nabla u_{\delta}-\nabla u\right)(1-t) d t d x .
\end{aligned}
$$

From Theorem 2.9 we have $I_{\delta}\left(u_{\delta}\right) \underset{\delta \rightarrow 0^{+}}{\longrightarrow} I(u), \delta \int_{\Omega}\left|\nabla u_{\delta}\right|^{2} d x \longrightarrow_{\delta \rightarrow 0^{+}} 0$, hence

$$
\begin{aligned}
& \lim _{\delta \rightarrow 0^{+}}\left\{\int_{\Omega} D G(\nabla u) \cdot\left(\nabla u_{\delta}-\nabla u\right) d x\right. \\
& \left.\quad+\int_{\Omega} \int_{0}^{1} D^{2} G\left((1-t) \nabla u+t \nabla u_{\delta}\right)\left(\nabla u_{\delta}-\nabla u, \nabla u_{\delta}-\nabla u\right)(1-t) d t d x\right\} \\
& =0 .
\end{aligned}
$$

On the other hand, minimality of $u$ implies

$$
\int_{\Omega} D G(\nabla u)\left(\nabla u_{\delta}-\nabla u\right) d x \geq 0 .
$$

Next we observe the estimate

$$
\begin{aligned}
& \int_{\Omega} \int_{0}^{1} D^{2} G\left((1-t) \nabla u+t \nabla u_{\delta}\right)\left(\nabla u_{\delta}-\nabla u, \nabla u_{\delta}-\nabla u\right)(1-t) d t d x \\
& \geq \int_{\Omega} \int_{0}^{1} \frac{\left|\nabla u_{\delta}-\nabla u\right|^{2}(1-t)}{1+\left|(1-t) \nabla u+t \nabla u_{\delta}\right|} d t d x .
\end{aligned}
$$

This implies that $\nabla u_{\delta} \rightarrow \nabla u$ a.e in $\Omega$ (possibly for some subsequence). Hence we get from (2.23) that

$$
\sqrt{1+\left|\nabla u_{\delta}\right|} \rightarrow \sqrt{1+|\nabla u|} \text { in } W_{2, \text { loc }}^{1}
$$

and the theorem is proved.

Remark 2.15. We mention that in $[\mathrm{FS}] \stackrel{\circ}{W} \stackrel{1}{L}{ }_{L n L}$ has a different definition but one of the equivalent characterizations of a function $u$ belonging to the space $\stackrel{\circ}{W} \stackrel{1}{L L n L}_{\text {is }}$ is that $u$ belongs to the Orlicz-Sobolev space generated by $A(t)=t \ln (1+t), t \geq 0$.

\section{LiNEARIZATION}

To study the obstacle problem, it is convenient to consider the variational inequality as an equation with a measure valued right-hand side and then 
to apply suitable methods in order to identify this measure. To this end, following $\left[F_{2}\right]$, we define

$$
w_{t}^{\varepsilon}:=u_{\delta}+t \eta h_{\varepsilon} \circ\left(u_{\delta}-\Phi\right)
$$

where $\delta \geq 0, u_{\delta}$ is given in Section $2, \eta \in C_{0}^{1}(\Omega), 0 \leq \eta \leq 1, t>0, \varepsilon>0, h_{\varepsilon} \in$ $C^{1}\left(R^{1}\right), 0 \leq h_{\varepsilon} \leq 1, h_{\varepsilon}=1$ on $(0, \varepsilon), h_{\varepsilon}=0$ on $(2 \varepsilon, \infty), h_{\varepsilon}^{\prime} \leq 0$. In case $\delta=0$ we have $u_{0}=u, u$ denoting the solution of (1.1). Then $w_{t}^{\varepsilon} \in \mathbb{K}^{*}$, if $\delta>0$, and $w_{t}^{\varepsilon} \in \mathbb{K}$ for $\delta=0$, hence

$$
\begin{aligned}
& \frac{1}{t}\left[\int_{\Omega} G_{\delta}\left(\nabla w_{t}^{\varepsilon}\right) d x-\int_{\Omega} G_{\delta}\left(\nabla u_{\delta}\right) d x\right] \geq 0 \Rightarrow(\text { as } t \rightarrow 0) \\
& \int_{\Omega} D G_{\delta}\left(\nabla u_{\delta}\right) \cdot \nabla\left(\eta h_{\varepsilon} \circ\left(u_{\delta}-\Phi\right)\right) d x \geq 0
\end{aligned}
$$

and there exists a Radon measure $\lambda$ (independent of $\varepsilon$ !) such that

$$
\int_{\Omega} D G_{\delta}\left(\nabla u_{\delta}\right) \cdot \nabla\left(\eta h_{\varepsilon} \circ\left(u_{\delta}-\Phi\right)\right) d x=\int_{\Omega} \eta d \lambda
$$

The fact that $\lambda$ does not depend on $\varepsilon$ can be seen by using $\tilde{w}=u_{\delta}+\eta t\left\{h_{\varepsilon} \circ\right.$ $\left.\left(u_{\delta}-\Phi\right)-h_{\varepsilon^{\prime}} \circ\left(u_{\delta}-\Phi\right)\right\}\left(\varepsilon<\varepsilon^{\prime}\right)$ as test function provided t is small enough.

Note that $(3.1)$ is valid for all small $\varepsilon>0$ and any $\eta \in C_{0}^{1}(\Omega)$. For estimating $\lambda$ we may therefore fix $\eta \geq 0$ and let $\varepsilon \rightarrow 0$, in order to get

$$
\begin{aligned}
\int_{\Omega} \eta d \lambda= & \int_{\Omega} D G_{\delta}\left(\nabla u_{\delta}\right) \cdot \nabla \eta h_{\varepsilon} \circ\left(u_{\delta}-\Phi\right) d x \\
& +\int_{\Omega} D G_{\delta}\left(\nabla u_{\delta}\right) \cdot \nabla\left(u_{\delta}-\Phi\right) h_{\varepsilon}^{\prime} \circ\left(u_{\delta}-\Phi\right) \eta d x \\
= & :(\alpha)+(\beta),
\end{aligned}
$$

where

$$
\begin{aligned}
(\beta)= & \int_{\Omega}\left\{D G_{\delta}\left(\nabla u_{\delta}\right)-D G_{\delta}(\nabla \Phi)\right\} \cdot \nabla\left(u_{\delta}-\Phi\right) h_{\varepsilon}^{\prime} \circ\left(u_{\delta}-\Phi\right) \eta d x \\
& +\int_{\Omega} D G_{\delta}(\nabla \Phi) \cdot \nabla\left(u_{\delta}-\Phi\right) h_{\varepsilon}^{\prime} \circ\left(u_{\delta}-\Phi\right) \eta d x \\
\leq & \int_{\Omega} D G_{\delta}(\nabla \Phi) \cdot \nabla\left(u_{\delta}-\Phi\right) h_{\varepsilon}^{\prime} \circ\left(u_{\delta}-\Phi\right) \eta d x \\
= & :(\gamma),
\end{aligned}
$$

and the estimate holds since $G_{\delta}$ is convex and $h_{\varepsilon}^{\prime} \leq 0$. We have

$$
\begin{aligned}
(\gamma)= & \int_{\Omega} D G_{\delta}(\nabla \Phi) \cdot \nabla\left(h_{\varepsilon} \circ\left(u_{\delta}-\Phi\right) \eta\right) d x \\
& -\int_{\Omega} D G_{\delta}(\nabla \Phi) h_{\varepsilon} \circ\left(u_{\delta}-\Phi\right) \cdot \nabla \eta d x \\
= & -\int_{\Omega} \operatorname{div}\left\{D G_{\delta}(\nabla \Phi)\right\} \eta h_{\varepsilon} \circ\left(u_{\delta}-\Phi\right) d x \\
& -\int_{\Omega} D G_{\delta}(\nabla \Phi) h_{\varepsilon} \circ\left(u_{\delta}-\Phi\right) \cdot \nabla \eta d x,
\end{aligned}
$$


which implies

$$
\begin{aligned}
& \int_{\Omega} \eta d \lambda \leq \int_{\Omega}\left\{D G_{\delta}\left(\nabla u_{\delta}\right)-D G_{\delta}(\nabla \Phi)\right\} \cdot \nabla \eta h_{\varepsilon} \circ\left(u_{\delta}-\Phi\right) d x \\
& \quad-\int_{\Omega} \operatorname{div}\left\{D G_{\delta}(\nabla \Phi)\right\} \eta h_{\varepsilon} \circ\left(u_{\delta}-\Phi\right) d x \\
& \underset{\varepsilon \rightarrow 0}{\longrightarrow} \int_{\left[u_{\delta}=\Phi\right]}\left\{D G_{\delta}\left(\nabla u_{\delta}\right)-D G_{\delta}(\nabla \Phi)\right\} \cdot \nabla \eta d x-\int_{\left[u_{\delta}=\Phi\right]} \operatorname{div}\left\{D G_{\delta}(\nabla \Phi)\right\} \eta d x .
\end{aligned}
$$

Since $\nabla u_{\delta}=\nabla \Phi$ a.e. on $\left[u_{\delta}=\Phi\right]$, we arrive at

$$
\int_{\Omega} \eta d \lambda \leq \int_{\Omega} \chi_{\left[u_{\delta}=\Phi\right]}\left(-\operatorname{div}\left\{D G_{\delta}(\nabla \Phi)\right\}\right) \eta d x .
$$

In particular, $\chi_{\left[u_{\delta}=\Phi\right]}\left(-\operatorname{div}\left\{D G_{\delta}(\nabla \Phi)\right\}\right) \geq 0$ a.e. and $\lambda$ takes the form

$$
\lambda=\lambda_{\delta}=\Theta_{\delta}\left(-\operatorname{div}\left\{D G_{\delta}(\nabla \Phi)\right\}\right) \times \text { Lebesgue measure }
$$

for some density $0 \leq \Theta_{\delta} \leq 1$ supported on $\left[u_{\delta}=\Phi\right]$.

Returning to (3.1) and observing that

$$
\int_{\Omega} D G_{\delta}\left(\nabla u_{\delta}\right) \cdot \nabla\left(\eta\left(1-h_{\varepsilon} \circ\left(u_{\delta}-\Phi\right)\right)\right) d x=0
$$

we get

$$
\int_{\Omega} D G_{\delta}\left(\nabla u_{\delta}\right) \cdot \nabla \eta d x=\int_{\Omega} \eta d \lambda_{\delta}
$$

Thus we have proved

Theorem 3.1. For any $\delta \geq 0$, there exists $f_{\delta} \in L^{\infty}(\Omega)$ such that $\left\|f_{\delta}\right\|_{\infty} \leq$ $\left\|\operatorname{div}\left\{D G_{\delta}(\nabla \Phi)\right\}\right\|_{\infty}$ and

$$
\int_{\Omega} D G_{\delta}\left(\nabla u_{\delta}\right) \cdot \nabla \varphi d x=\int_{\Omega} f_{\delta} \varphi d x, \forall \varphi \in C_{0}^{1}(\Omega) .
$$

In particular, $\left\|f_{\delta}\right\|_{\infty}$ is bounded independently of $\delta$.

Remark 3.2. The proof of Theorem 3.1 given before does not use the fact that $u_{\delta}$ is of class $W_{2, \text { loc }}^{2}(\Omega)$. The higher differentiability of $u_{\delta}$ allows to perform an integration by parts in formula (3.1), and afetr passing to the limit $\epsilon \downarrow 0$ we immediately deduce the representation of the measure $\lambda$.

\section{A Caccioppoli-type inequality}

In this section we prove the following Caccioppoli-type inequality. 
Theorem 4.1. Let $u$ be the minimizer from Theorem 1.1. Then, for arbitrary balls $B_{r}\left(x_{0}\right) \subset B_{R}\left(x_{0}\right) \subset \Omega$, we have the estimate

$$
\begin{aligned}
\int_{B_{r}\left(x_{0}\right)} \mid \nabla & \sqrt{1+\left.|\nabla u|\right|^{2}} d x \\
\leq & C\left\{\frac{1}{(R-r)^{2}} \int_{B_{R}\left(x_{0}\right)} \frac{\ln (1+|\nabla u|)}{|\nabla u|}|\nabla u-X| d x\right. \\
& \left.+\int_{B_{R}\left(x_{0}\right)}(1+|\nabla u|) d x+\frac{1}{R-r} \int_{B_{R}\left(x_{0}\right)}|\nabla u-X| d x\right\},
\end{aligned}
$$

where $X$ is any vector in $\mathbb{R}^{n}, C=C(n, \Phi)$.

Proof. Let $u_{\delta}$ be as in the previous sections. Using $u_{\delta} \in W_{2, \text { loc }}^{2}(\Omega)$ for $\delta>0$ (which will be assumed from now on), we get from Theorem 3.1 that

$$
\int_{\Omega} D^{2} G_{\delta}\left(\nabla u_{\delta}\right)\left(\partial_{\gamma} \nabla u_{\delta}, \nabla \varphi\right) d x=-\int_{\Omega} f_{\delta} \partial_{\gamma} \varphi d x
$$

Let $\varphi=\eta^{2}\left(\partial_{\gamma} u_{\delta}-X_{\gamma}\right)$ for $\eta \in C_{0}^{1}(\Omega), 0 \leq \eta \leq 1, X \in \mathbb{R}^{n}$.

Using summation over $\gamma$ we deduce

$$
\begin{gathered}
\int_{\Omega} \eta^{2} D^{2} G_{\delta}\left(\nabla u_{\delta}\right)\left(\partial_{\gamma} \nabla u_{\delta}, \partial_{\gamma} \nabla u_{\delta}\right) d x= \\
-\int_{\Omega} 2 D^{2} G_{\delta}\left(\nabla u_{\delta}\right)\left(\partial_{\gamma} \nabla u_{\delta}, \nabla \eta\left[\partial_{\gamma} u_{\delta}-X_{\gamma}\right]\right) \eta d x-\int_{\Omega} f_{\delta} \partial_{\gamma}\left(\eta^{2}\left[\partial_{\gamma} u_{\delta}-X_{\gamma}\right]\right) d x,
\end{gathered}
$$

and as in the proof of Lemma 3.1 in $[\mathrm{FS}]$ we get the estimate

$$
\begin{aligned}
& \int_{\Omega} \eta^{2}\left[\delta\left|\nabla^{2} u_{\delta}\right|^{2}+\left|\nabla \sqrt{1+\mid \nabla u_{\delta}}\right|^{2}\right] d x \\
\leq & C(n)\left\{\|\nabla \eta\|_{\infty} \int_{s t p \eta}\left[\delta\left|\nabla u_{\delta}-X\right|^{2}+\frac{\ln \left(1+\left|\nabla u_{\delta}\right|\right)}{\left|\nabla u_{\delta}\right|}\left|\nabla u_{\delta}-X\right|^{2}\right] d x\right. \\
& \left.+\int_{\Omega}\left|f_{\delta}\right|\left|\nabla\left(\eta^{2}\left[\nabla u_{\delta}-X\right]\right)\right| d x\right\} .
\end{aligned}
$$

We recall $\left\|f_{\delta}\right\|_{\infty} \leq$ const. independent of $\delta$ and observe

$$
\int_{\Omega}\left|\nabla\left(\eta^{2}\left[\nabla u_{\delta}-X\right]\right)\right| d x \leq C\left\{\int_{\Omega} \eta^{2}\left|\nabla^{2} u_{\delta}\right| d x+\int_{s p t \eta}\|\nabla \eta\|_{\infty}\left|\nabla u_{\delta}-X\right| d x\right\}
$$

and

$$
\int_{\Omega} \eta^{2}\left|\nabla^{2} u_{\delta}\right| d x \leq \varepsilon \int_{\Omega} \eta^{2} \frac{1}{1+\left|\nabla u_{\delta}\right|}\left|\nabla^{2} u_{\delta}\right|^{2} d x+\frac{1}{\varepsilon} \int_{\Omega} \eta^{2}\left(1+\left|\nabla u_{\delta}\right|\right) d x .
$$

Of course, inequality (4.2) remains valid if the left-hand side is replaced by

$$
\int_{\Omega} \eta^{2} D^{2} G_{\delta}\left(\nabla u_{\delta}\right)\left(\partial_{\gamma} \nabla u_{\delta}, \partial_{\gamma} \nabla u_{\delta}\right) d x
$$

On the other hand,

$$
\frac{1}{1+|\nabla u|}\left|D^{2} u_{\delta}\right|^{2} \leq D^{2} G_{\delta}\left(\nabla u_{\delta}\right)\left(\partial_{\gamma} \nabla u_{\delta}, \partial_{\gamma} \nabla u_{\delta}\right)
$$


and by choosing $\varepsilon>0$ small enough, we may absorb $\varepsilon \int_{\Omega} \eta^{2} \frac{1}{1+|\nabla u|}\left|D^{2} u_{\delta}\right|^{2} d x$ into the left-hand side. This finally implies

$$
\begin{aligned}
& \int_{\Omega} \eta^{2}\left|\nabla \sqrt{1+\left|\nabla u_{\delta}\right|}\right|^{2} d x
\end{aligned}
$$

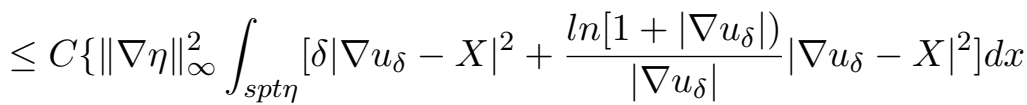

$$
\begin{aligned}
& \left.+\int_{s p t \eta}\left(1+\left|\nabla u_{\delta}\right|\right) d x+\|\nabla \eta\|_{\infty} \int_{s p t \eta}\left|\nabla u_{\delta}-X\right| d x\right\} .
\end{aligned}
$$

Using the imbedding theorem as in [FS], we may now pass to the limit $\delta \rightarrow 0$ in the above inequality which finishes the proof of the theorem.

\section{Blow-Up: PROOF OF PARTial REgUlarity}

We fix some $0<\mu<1$ and denote by $u$ the solution to the obstacle problem from Theorem 1.1. Let us further assume that $n \leq 4$. We have the following

Lemma 5.1. Fix some $L>0$ and calculate $C_{0}=C_{0}(n, L)$ as indicated in the proof. Then, for all $\tau \in(0,1)$, we find a number $\varepsilon=\varepsilon(n, \tau, L)$ such that

$$
(|\nabla u|)_{x_{0}, R}<L \text { and } f_{B_{R}\left(x_{0}\right)}\left|\nabla u-(\nabla u)_{x_{0}, R}\right|^{2} d x+R^{2 \mu}<\varepsilon^{2}
$$

imply

$$
f_{B_{\tau R}\left(x_{0}\right)}\left|\nabla u-(\nabla u)_{x_{0}, \tau R}\right|^{2} d x \leq C_{0} \tau^{2}\left\{f_{B_{R}\left(x_{0}\right)}\left|\nabla u-(\nabla u)_{x_{0}, R}\right|^{2}+R^{2 \mu}\right\}
$$

for any ball $B_{R}\left(x_{0}\right) \subset \Omega$.

Here $(g)_{y, \rho}$ denotes the mean value $f_{B_{\rho}(y)} g d x$. In the formulation of the Lemma we replaced the "standard assumption" $\left|(\nabla u)_{x_{0}, R}\right|<L$ by a slightly stronger one.

Lemma 5.1 will be proved at the end of this section. We first show our main result Theorem 1.1.

Let us introduce the set

$\Omega_{0}=\left\{x \in \Omega: x\right.$ is a Lebesgue point for $\nabla u$ and $|\nabla u|$ and $f_{B_{r}\left(x_{0}\right)} \mid \nabla u-$ $\left.\left.(\nabla u)_{x_{0}, r}\right|^{2} d x \longrightarrow_{r \downarrow 0} 0\right\}$.

Clearly $\left|\Omega-\Omega_{0}\right|=0$. In order to prove the theorem with the help of Lemma 5.1 , we need only to show that any point $x_{0}$ from $\Omega_{0}$ has some neighborhood in $\Omega$ on which $\nabla u$ is Hölder continuous.

Let $x_{0} \in \Omega$ and let

$$
\begin{gathered}
L:=\max \left\{2|\nabla u|\left(x_{0}\right), 1\right\}, \\
|\nabla u|\left(x_{0}\right)=\lim _{r \rightarrow 0} f_{B_{r}\left(x_{0}\right)}|\nabla u| d x .
\end{gathered}
$$


This determines the constant $C_{0}=C_{0}(L)$. Fix $\tau$ such that

$$
C_{0} \tau^{2}=\frac{1}{2}
$$

and calculate $\varepsilon$ w.r.t. this data. Note the inequality

$$
(|\nabla u|)_{x_{0}, \tau^{k+1} R} \leq \tau^{-\frac{n}{2}} \sum_{i=0}^{k} E\left(x_{0}, \tau^{i} R\right)^{\frac{1}{2}}+(|\nabla u|)_{x_{0}, R}
$$

being valid for any $R$ such that $B_{R}\left(x_{0}\right) \subset \Omega$ and any $k \in \mathbf{N}$. Here $E\left(x_{0}, R\right):=f_{B_{R}\left(x_{0}\right)}\left|\nabla u-(\nabla u)_{x_{0}, R}\right|^{2} d x$. Let us further set $\theta=\tau^{2 \mu}$ (w.l.o.g. $\left.\theta<\frac{1}{2}\right)$.

A number $\bar{\varepsilon}$ is chosen according to

$$
\left\{\begin{array}{l}
\tau^{-\frac{n}{2}} \sum_{i=0}^{\infty} 2^{-\frac{i}{2}} \frac{1}{\sqrt{1-2 \theta}} \bar{\varepsilon}<L / 3 \\
\bar{\varepsilon}^{2} \leq \min \left\{\frac{1}{4}, \frac{1-2 \theta}{2}\right\} \varepsilon^{2}
\end{array} .\right.
$$

Finally, we fix $R>0$ such that

$$
E\left(x_{0}, R\right)+R^{2 \mu}<\bar{\varepsilon}^{2}, \quad(|\nabla u|)_{x_{0}, R}<\frac{2}{3} L .
$$

Proposition 5.2. For any $k \in N$ we have

$$
E\left(x_{0}, \tau^{k} R\right) \leq 2^{-k} E\left(x_{0}, R\right)+\sum_{j=1}^{k} 2^{-j} \theta^{k-j} R^{2 \mu}
$$

Proof. We prove the proposition by induction. Let $k=1$. Then (5.6) implies (5.2). Hence (5.3) holds. Thus, by the choice of $\tau$,

$$
E\left(x_{0}, \tau R\right) \leq \frac{1}{2}\left(E\left(x_{0}, R\right)+R^{2 \mu}\right)
$$

and $(5.7)_{1}$ holds. 
Assume now $(5.7)_{k}$. Then

$$
\begin{aligned}
& E\left(x_{0}, \tau^{k} R\right) \leq 2^{-k} E\left(x_{0}, R\right)+\theta^{k} \sum_{j=1}^{k}\left(\frac{1}{2 \theta}\right)^{j} R^{2 \mu} \\
& =2^{-k} E\left(x_{0}, R\right)+\theta^{k}\left(\frac{1-\left(\frac{1}{2 \theta}\right)^{k+1}}{1-\frac{1}{2 \theta}}-1\right) R^{2 \mu} \\
& =2^{-k} E\left(x_{0}, R\right)+\theta^{k} \frac{\frac{1}{2 \theta}-\left(\frac{1}{2 \theta}\right)^{k+1}}{1-\frac{1}{2 \theta}} R^{2 \mu} \\
& =2^{-k} E\left(x_{0}, R\right)+\frac{\frac{1}{2} \theta^{k-1}-\frac{1}{\theta} 2^{-k-1}}{1-\frac{1}{2 \theta}} R^{2 \mu} \\
& =2^{-k} E\left(x_{0}, R\right)+\frac{\theta^{k}-2^{-k}}{2 \theta-1} R^{2 \mu} \\
& =2^{-k} E\left(x_{0}, R\right)+\frac{2^{-k}-\theta^{k}}{1-2 \theta} R^{2 \mu} \\
& \leq 2^{-k}\left\{E\left(x_{0}, R\right)+\frac{1}{1-2 \theta} R^{2 \mu}\right\} \\
& \leq \frac{2^{-k}}{1-2 \theta} \bar{\varepsilon}^{2},
\end{aligned}
$$

hence

$$
\begin{gathered}
E\left(x_{0}, \tau^{k} R\right)+\left(\tau^{k} R\right)^{2 \mu} \leq \frac{2^{-k}}{1-2 \theta} \bar{\varepsilon}^{2}+\tau^{k 2 \mu} R^{2 \mu} \leq \frac{2}{1-2 \theta} \bar{\varepsilon}^{2} \leq \varepsilon^{2}, \text { i.e. } \\
E\left(x_{0}, \tau^{k} R\right)+\left(\tau^{k} R\right)^{2 \mu}<\varepsilon^{2} .
\end{gathered}
$$

Next, we have

$$
(|\nabla u|)_{x_{0}, \tau^{k} R} \leq \tau^{-\frac{n}{2}} \sum_{i=1}^{k-1} E\left(x_{0}, \tau^{i} R\right)^{\frac{1}{2}}+(|\nabla u|)_{x_{0}, R},
$$

and since we may assume that $(5.7)_{j}$ holds for any $j \leq k$, we get

$$
(|\nabla u|)_{x_{0}, \tau^{k} R} \leq \tau^{-\frac{n}{2}} \sum_{i=1}^{k-1}\left[2^{-i}\left\{E\left(x_{0}, R\right)+\frac{1}{1-2 \theta} R^{2 \mu}\right\}\right]^{\frac{1}{2}}+(|\nabla u|)_{x_{0}, R}
$$

which is a consequence of (5.8).

Therefore

$$
\begin{aligned}
(|\nabla u|)_{x_{0}, \tau^{k} R} & \leq \tau^{-\frac{n}{2}} \sum_{i=0}^{\infty} 2^{-\frac{i}{2}} \frac{1}{\sqrt{1-2 \theta}}\left(E\left(x_{0}, R\right)+R^{2 \mu}\right)^{\frac{1}{2}}+(|\nabla u|)_{x_{0}, R} \\
& \leq \tau^{-\frac{n}{2}} \bar{\varepsilon} \frac{1}{\sqrt{1-2 \theta}} \sum_{i=0}^{\infty} 2^{-\frac{i}{2}}+(|\nabla u|)_{x_{0}, R} \\
& <\frac{L}{3}+(|\nabla u|)_{x_{0}, R} \leq L
\end{aligned}
$$


From this inequality and (5.9) we see that Lemma 5.1 can be applied. Thus,

$$
\begin{aligned}
E\left(x_{0}, \tau^{k+1} R\right) & \leq \frac{1}{2}\left(E\left(x_{0}, \tau^{k} R\right)+\left(\tau^{k} R\right)^{2 \mu}\right) \\
& \leq \frac{1}{2}\left(2^{-k} E\left(x_{0}, R\right)+\sum_{j=1}^{k} 2^{-j} \theta^{k-j} R^{2 \mu}\right)+\frac{1}{2} \theta^{k} R^{2 \mu} \\
& =2^{-k-1} E\left(x_{0}, R\right)+\left\{\sum_{j=1}^{k} 2^{-(j+1)} \theta^{(k+1)-(j+1)}+\frac{1}{2} \theta^{k}\right\} R^{2 \mu} \\
& =2^{-k-1} E\left(x_{0}, R\right)+\sum_{j=1}^{k+1} 2^{-j} \theta^{k+1-j} R^{2 \mu}
\end{aligned}
$$

and the proof of the proposition is complete.

As shown in (5.8), we get from $(5.7)_{k}$

$$
E\left(x_{0}, \tau^{k} R\right) \leq 2^{-k} \frac{1}{1-2 \theta}\left\{E\left(x_{0}, R\right)+R^{2 \mu}\right\}, \quad \forall k \in N
$$

which turns into the inequality

$$
E\left(x_{0}, r\right) \leq \operatorname{const} .\left(\frac{r}{R}\right)^{\alpha}\left\{E\left(x_{0}, R\right)+R^{2 \mu}\right\}, \quad \forall r<R
$$

for some exponent $\alpha>0$.

Clearly, (5.6) implies

$$
E(y, R)+R^{2 \mu}<\bar{\varepsilon}^{2}, \quad(|\nabla u|)_{y, R}<\frac{2}{3} L
$$

for any $y$ near $x_{0}$ with $R$ fixed, hence (5.10), (5.11) also hold at $y$ which means that $\nabla u$ is Hölder continuous for example in $B_{R / 2}\left(x_{0}\right)$ with some exponent $\alpha$. Hölder continuity near $x_{0}$ with any exponent $<\mu$ can be seen by choosing $\tau$ in $a$ different way.

Proof of Lemma 5.1. We follow the proof of Lemma 4.1 in [FS] and argue by contradiction assuming

$$
\left\{\begin{array}{l}
(|\nabla u|)_{x_{k}, R_{k}} \leq L \quad A_{k}:=(\nabla u)_{x_{0}, R_{k}} \\
E\left(u, B_{R_{k}}\left(x_{k}\right)\right)+R_{k}^{2 \mu}=\varepsilon_{k}^{2} \rightarrow 0 \\
E\left(u, B_{\tau R_{k}}\left(x_{k}\right)\right)>C_{0} \tau^{2} \varepsilon_{k}^{2}
\end{array}\right.
$$

for a sequence $B_{R_{k}}\left(x_{k}\right)$. Let

$$
\begin{gathered}
v_{k}(z):=\frac{1}{\varepsilon_{k} R_{k}}\left(u\left(x_{k}+R_{k} z\right)-a_{k}-R_{k} A_{k} z\right), z \in B_{1}, \\
a_{k}:=f_{B_{R_{k}}\left(x_{0}\right)} u d x .
\end{gathered}
$$

Then, for suitable $A \in R^{n}$ and $v \in W_{2}^{1}\left(B_{1}\right)$, we get

$A_{k} \rightarrow A, v_{k} \rightarrow v$ strongly in $L^{2}\left(B_{1}\right)$,

$\nabla v_{k} \rightarrow \nabla v$ weakly in $L^{2}\left(B_{1}, R^{n}\right)$ 
$\varepsilon_{k} \nabla v_{k} \rightarrow 0$ strongly in $L^{2}\left(B_{1}, R^{n}\right)$ and a.e.

which is true at least for some subsequence. From Theorem 3.1, we know

$$
\int_{\Omega} D G(\nabla u) \cdot \nabla \psi d x=\int_{\Omega} f \psi d x, \forall \psi \in C_{0}^{1}(\Omega) .
$$

Let $\varphi \in C_{0}^{1}\left(B_{1}\right)$ and define $\psi(x)=\varphi\left(\frac{x-x_{k}}{R_{k}}\right), x \in B_{R_{k}}\left(x_{k}\right)$. Then the above equation reads

$$
R_{k}^{n-1} \int_{B_{1}} D G\left(\varepsilon_{k} \nabla v_{k}+A_{k}\right) \cdot \nabla \varphi d z=R_{k}^{n} \int_{B_{1}} \varphi(z) f\left(R_{k} z+x_{k}\right) d z
$$

which implies

$$
\int_{B_{1}} \frac{1}{\varepsilon_{k}}\left\{D G\left(\varepsilon_{k} \nabla v_{k}+A_{k}\right)-D G\left(A_{k}\right)\right\} \cdot \nabla \varphi d z=\frac{R_{k}}{\varepsilon_{k}} \int_{B_{1}} \varphi(z) f\left(R_{k} z+x_{k}\right) d z
$$

Using $R_{k} / \varepsilon_{k} \rightarrow 0$ and the boundedness of $f$ we get

$$
\lim _{k \rightarrow \infty} \int_{B_{1}} \frac{1}{\varepsilon_{k}}\left\{D G\left(\varepsilon_{k} \nabla v_{k}+A_{k}\right)-D G\left(A_{k}\right)\right\} \cdot \nabla \varphi d z=0
$$

hence

$$
\int_{B_{1}} D^{2} G(A)(\nabla v, \nabla \varphi) d z=0
$$

which is an elliptic equation with constant coefficients.

Thus there exists $C_{1}=C_{1}(L)$ such that

$$
f_{B_{\tau}}\left|\nabla v-(\nabla v)_{\tau}\right|^{2} d z \leq C_{1} \tau^{2} f_{B_{1}}|\nabla v|^{2} d z
$$

Letting $C_{0}=2 C_{1}$, the foregoing inequality will contradict our assumption (5.12) as soon as we can establish

$$
\nabla v_{k} \rightarrow \nabla v \text { strongly in } L_{\text {loc }}^{2}\left(B_{1}, R^{n}\right) .
$$

To this end, we argue in two steps.

Step 1: Let $\varphi_{k}(z)=\frac{1}{\varepsilon_{k}}\left(\sqrt{1+\left|\varepsilon_{k} \nabla v_{k}+A_{k}\right|}-\sqrt{1+\left|A_{k}\right|}\right), z \in B_{1}$. Then $\left|\varphi_{k}\right| \leq \frac{1}{2}\left|\nabla v_{k}\right|$ and since $\left\{\nabla v_{k}\right\}$ is uniformly bounded in $L^{2}\left(B_{1}, R^{n}\right)$ we see that

$$
\sup \int_{B_{1}} \varphi_{k}^{2} d z<\infty
$$

Next we observe

$$
\begin{aligned}
\nabla \varphi_{k}(z) & =\frac{1}{\varepsilon_{k}} \nabla \sqrt{1+\left|\varepsilon_{k} \nabla v_{k}+A_{k}\right|}=\frac{1}{\varepsilon_{k}} \nabla \sqrt{1+|\nabla u|\left(x_{k}+R_{k} z\right)} \\
& =\frac{R_{k}}{\varepsilon_{k}}(\nabla \sqrt{1+|\nabla u|})\left(x_{k}+R_{k} z\right)
\end{aligned}
$$


so that

$$
\begin{aligned}
\int_{B_{1}}\left|\nabla \varphi_{k}\right|^{2} d z & =\frac{R_{k}^{2}}{\varepsilon_{k}^{2}} \int_{B_{1}}|\nabla \sqrt{1+|\nabla u|}|^{2}\left(x_{k}+R_{k} z\right) d z \\
& =\varepsilon_{k}^{-2} R_{k}^{2-n} \int_{B_{R_{k}}\left(x_{k}\right)}|\nabla \sqrt{1+|\nabla u|}|^{2} d x
\end{aligned}
$$

and in the same manner for $0<t<1$

$$
\int_{B_{t}}\left|\nabla \varphi_{k}\right|^{2} d z \leq \varepsilon_{k}^{-2} R_{k}^{2-n} \int_{B_{t R_{k}}\left(x_{k}\right)}|\nabla \sqrt{1+|\nabla u|}|^{2} d x .
$$

Theorem 4.1 gives

$$
\begin{aligned}
& \int_{B_{t}}\left|\nabla \varphi_{k}\right|^{2} d z \\
& \leq C\left\{\varepsilon_{k}^{-2} R_{k}^{2-n}(1-t)^{-2} R_{k}^{-2} \int_{B_{R_{k}}\left(x_{k}\right)} \frac{\ln (1+|\nabla u|)}{|\nabla u|}\left|\nabla u-A_{k}\right|^{2} d x\right. \\
& \quad+\varepsilon_{k}^{-2} R_{k}^{2-n} \int_{B_{R_{k}}\left(x_{k}\right)}(1+|\nabla u|) d x \\
& \left.\quad+\varepsilon_{k}^{-2} R_{k}^{2-n}(1-t)^{-1} R_{k}^{-1} \int_{B_{R_{k}}\left(x_{k}\right)}\left|\nabla u-A_{k}\right| d x\right\} .
\end{aligned}
$$

Clearly,

$$
\begin{aligned}
& \varepsilon_{k}^{-2} R_{k}^{2-n}(1-t)^{-2} R_{k}^{-2} \int_{B_{R_{k}}\left(x_{k}\right)} \frac{\ln (1+|\nabla u|)}{|\nabla u|}\left|\nabla u-A_{k}\right|^{2} d x \\
\leq & C(1-t)^{-2} \varepsilon_{k}^{-2} f_{B_{k}\left(x_{k}\right)}\left|\nabla u-A_{k}\right|^{2} d x \leq C(1-t)^{-2},
\end{aligned}
$$

and

$$
\begin{aligned}
\varepsilon_{k}^{-2} R_{k}^{2-n} \int_{B_{R_{k}}\left(x_{k}\right)}(1+|\nabla u|) d x & =c_{n} \frac{R_{k}^{2}}{\varepsilon_{k}^{2}}+\frac{R_{k}^{2}}{\varepsilon_{k}^{2}} f_{B_{R_{k}}\left(x_{k}\right)}|\nabla u| d x \\
& \leq\left(c_{n}+L\right) \frac{R_{k}^{2}}{\varepsilon_{k}^{2}} \rightarrow 0 \text { as } k \rightarrow \infty,
\end{aligned}
$$

where we have used our assumption $f_{B_{R_{k}}\left(x_{k}\right)}|\nabla u| d x<L$.

On the other hand,

$$
\begin{aligned}
& \varepsilon_{k}^{-2} R_{k}^{2-n}(1-t)^{-1} R_{k}^{-1} \int_{B_{R_{k}}\left(x_{k}\right)}\left|\nabla u-A_{k}\right| d x \\
\leq & \varepsilon_{k}^{-2} R_{k}^{2-n}(1-t)^{-1} R_{k}^{-1}\left(\int_{B_{R_{k}}\left(x_{k}\right)}\left|\nabla u-A_{k}\right|^{2} d x\right)^{\frac{1}{2}} R_{k}^{\frac{n}{2}} \\
\leq & \varepsilon_{k}^{-2} R_{k}^{2-n}(1-t)^{-1} R_{k}^{-1} \varepsilon_{k} R_{k}^{\frac{n}{2}} R_{k}^{\frac{n}{2}} \\
= & (1-t)^{-1} \varepsilon_{k}^{-1} R_{k} \rightarrow 0 \text { as } k \rightarrow \infty .
\end{aligned}
$$


Hence we have established

$$
\sup _{k}\left\|\varphi_{k}\right\|_{W^{1,2}\left(B_{t}\right)}<\infty, \quad \forall 0<t<1 .
$$

For some large number $\mathrm{M}$ (depending on $\mathrm{L}$ ) we have

$$
\varphi_{k} \geq \frac{1}{2} \frac{1}{\varepsilon_{k}} \sqrt{\varepsilon_{k}\left|\nabla v_{k}\right|} \text { on } B_{t} \cap\left[\varepsilon_{k}\left|\nabla v_{k}\right|>M\right]
$$

so that

$$
\int_{B_{t} \cap\left[\varepsilon_{k}\left|\nabla v_{k}\right|>M\right]}\left|\nabla v_{k}\right|^{2} d z \leq 2^{4} \varepsilon_{k}^{2} \int_{B_{t}}\left|\varphi_{k}\right|^{4} d z .
$$

By (5.14) and the embedding theorem we deduce $(n \leq 4$ !)

$$
\int_{B_{t} \cap\left[\varepsilon_{k}\left|\nabla v_{k}\right|>M\right]}\left|\nabla v_{k}\right|^{2} d z \rightarrow 0 \text { as } k \rightarrow \infty
$$

and $v \in C^{\infty}\left(B_{1}\right)$ obviously implies

$$
\int_{B_{t} \cap\left[\varepsilon_{k}\left|\nabla v_{k}\right|>M\right]}\left|\nabla v_{k}-\nabla v\right|^{2} d z \rightarrow 0 \quad \text { as } k \rightarrow \infty .
$$

Step 2: Discussion of $\int_{B_{t} \cap\left[\varepsilon_{k}\left|\nabla v_{k}\right|<M\right]}\left|\nabla v_{k}\right|^{2} d z$.

Here we follow again [FS]. Consider $\varphi \in C_{0}^{1}\left(B_{1}\right), \varphi \geq 0$, and observe

$$
\begin{aligned}
& \varepsilon_{k}^{2} \int_{B_{1}} \int_{0}^{1} \varphi D^{2} G\left(A_{k}+\varepsilon_{k} \nabla v+s \varepsilon_{k}\left(\nabla v_{k}-\nabla v\right)\right) \\
& \cdot\left(\nabla v_{k}-\nabla v, \nabla v_{k}-\nabla v\right)(1-s) d s d z \\
= & \int_{B_{1}} \varphi\left[G\left(A_{k}+\varepsilon_{k}+\varepsilon_{k} \nabla v_{k}\right)-G\left(A_{k}+\varepsilon_{k} \nabla v\right)\right] d z \\
& -\int_{B_{1}} \varepsilon_{k} \varphi D G\left(A_{k}+\varepsilon_{k} \nabla v\right) \cdot\left(\nabla v_{k}-\nabla v\right) d z .
\end{aligned}
$$

The right hand side of (5.16) equals

$$
\begin{aligned}
& \int_{B_{1}} G\left(A_{k}+\varepsilon_{k} \nabla v_{k}\right) d z-\int_{B_{1}}\left\{(1-\varphi) G\left(A_{k}+\varepsilon_{k} \nabla v_{k}\right)+\varphi G\left(A_{k}+\varepsilon_{k} \nabla v\right)\right\} d z \\
& \quad-\int_{B_{1}} \varepsilon_{k} \varphi D G\left(A_{k}+\varepsilon_{k} \nabla v\right) \cdot\left(\nabla v_{k}-\nabla v\right) d z \\
& \leq \int_{B_{1}} G\left(A_{k}+\varepsilon_{k} \nabla v_{k}\right) d z-\int_{B_{1}} G\left(A_{k}+\varepsilon_{k}\left[\varphi \nabla v+(1-\varphi) \nabla v_{k}\right]\right) d z \\
& \quad-\int_{B_{1}} \varepsilon_{k} D G\left(A_{k}+\varepsilon_{k} \nabla v\right) \cdot\left(\nabla v_{k}-\nabla v\right) d z .
\end{aligned}
$$

By Theorem $3.1 \mathrm{u}$ is the minimizer of $w \longmapsto \int_{\Omega}[G(\nabla w)-f w] d x$ in $\stackrel{\circ}{W}_{A}^{1}(\Omega)$. After transformation and after dropping constants from the functional we see that $v_{k}$ minimizes

$$
h \longmapsto \int_{B_{1}}\left\{G\left(\varepsilon_{k} \nabla h+A_{k}\right)-\varepsilon_{k} R_{k} f h\right\} d z
$$


on $B_{1}$ w.r.t. its boundary values, i.e.,

$$
\begin{aligned}
\int_{B_{1}} G\left(\varepsilon_{k} \nabla v_{k}+A_{k}\right) d z \leq & \int_{B_{1}} G\left(\varepsilon_{k} \nabla\left[v_{k}+\varphi\left(v-v_{k}\right)\right]+A_{k}\right) d z \\
& +\int_{B_{1}} \varepsilon_{k} R_{k} f \varphi\left(v_{k}-v\right) d z .
\end{aligned}
$$

The last integral is the only new term which occurs compared to the calculations in [FS].

We therefore get, as in [FS], from (5.16)

$$
\begin{aligned}
& \int_{B_{1}} \int_{0}^{1} \varphi D^{2} G\left(A_{k}+\varepsilon_{k} \nabla v+s \varepsilon_{k}\left(\nabla v_{k}-\nabla v\right)\right) \\
& \cdot\left(\nabla v_{k}-\nabla v, \nabla v_{k}-\nabla v\right)(1-s) d s d z \\
\leq & o(1)+\frac{R_{k}}{\varepsilon_{k}} \int_{B_{1}} f \varphi\left(v_{k}-v\right) d z \rightarrow 0 \text { as } k \rightarrow+\infty .
\end{aligned}
$$

Using now the bounds of $D^{2} G$ we find

$$
\lim _{k \rightarrow \infty} \int_{B_{1}} \varphi\left|\nabla v_{k}-\nabla v\right|^{2}\left(1+\left|A_{k}\right|+\varepsilon_{k}|\nabla v|+\varepsilon_{k}\left|\nabla v_{k}-\nabla v\right|\right)^{-1} d z=0 .
$$

In particular, choosing $\varphi=1$ on $B_{t}$,

$$
\lim _{k \rightarrow+\infty} \int_{B_{t} \cap\left[\varepsilon_{k}\left|\nabla v_{k}\right|<M\right]}\left|\nabla v_{k}-\nabla v\right|^{2} d z=0
$$

This together with (5.15) implies (5.13), the proof of the Lemma is complete.

Thus Theorem 1.1 is proved. For proving Theorem 1.2, we modify the foregoing argument following the lines of [FO] with obvious modifications. The details are left to the reader.

Acknowledgment. Part of this paper was written during the first author's stay in China during March 1997. The first author would like to thank the Wuhan Institute of Physics and Mathematics, Chinese Academy of Sciences, for showing kind hospitality. The second author was partially supported by NSFC and the Academy of Finland.

\section{REFERENCES}

[BAH $]$ R. Bird, R. Armstrong and O. Hassager, Dynamics of polymeric liquids, Vol. 1, Fluid mechanics, John Wiley, 1987.

$\left[\mathrm{F}_{1}\right] \quad$ M. Fuchs, Topics in the Calculus of Variations, Vieweg,1994.

$\left[\mathrm{F}_{2}\right] \quad$ M. Fuchs, Hölder continuity of the gradient for degenerate variational inequalities, Nonl. Analysis, 15 (1990), 85-100.

[FO] M. Fuchs and V. Osmolovski, Variational integrals on Orlicz-Sobolev spaces. J. Anal. Appl. (Z.A.A.), 17 (1998), No. 2, 393-415.

[FS] M. Fuchs and G. Seregin, A regularity theory for variational integrals with LLnLgrowth, Preprint No. 471, SFB 256, Univ. Bonn, Calc. Var. Partial Differential Equations. 6 (1998) 2, 171-187. 
[HKM] J. Heinonen, T. Kilpeläinen and O.Martio, Nonlinear potential theory of degenerate elliptic equations, Clarendon Press,1993.

[KR] M. A. Krasnoselskii and J. B. Rutickii, Convex functions and Orlicz spaces, Noordhof, Groningen, 1961.

[KS] D. Kinderlehrer and G.Stampacchia, Variational inequalities and their applications, Acad. Press, San Diego, 1980.

MARTIN FUCHS

UNIVERSitÄT DES SAARLANDES

FACHBEREICH 9 MATHEMATIK

POSTFACH 151150

D-66041 SaARBrüCKEn, GERMANY

E-mail address: fuchs@math.uni-sb.de

Li GONGBAO

Wuhan Institute of Physics and Mathematics

Chinese Academy of Sciences

P.O. Box 71010, PEOPLE'S REPUBLIC OF CHINA

E-mail address: ligb@math.whcnc.ac.cn 


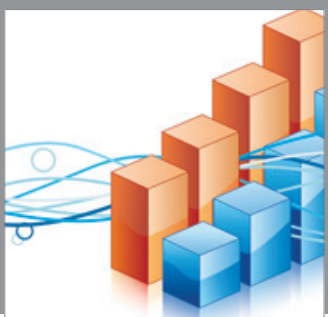

Advances in

Operations Research

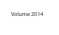

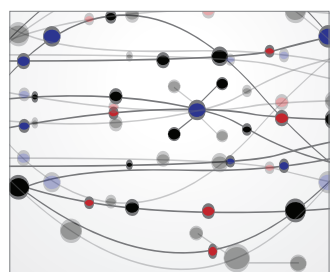

\section{The Scientific} World Journal
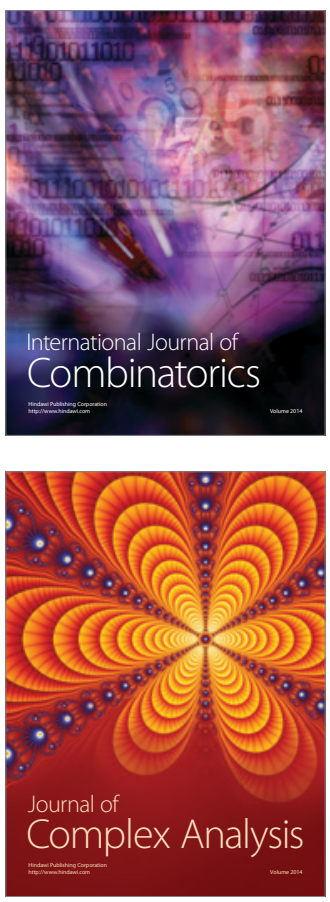

International Journal of

Mathematics and

Mathematical

Sciences
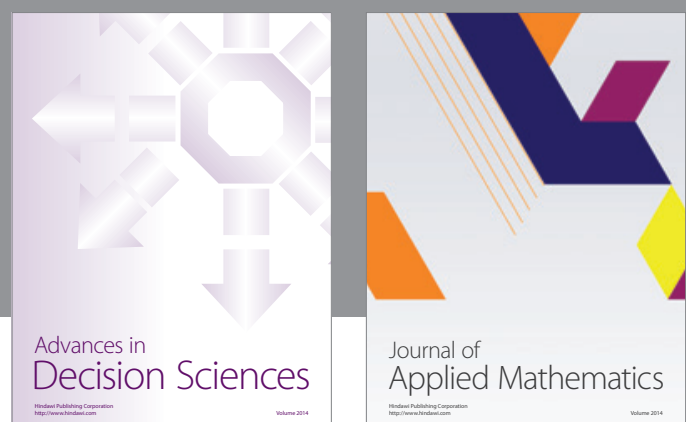

Journal of

Applied Mathematics
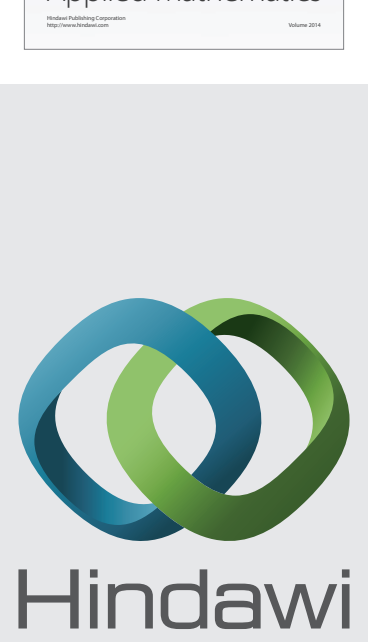

Submit your manuscripts at http://www.hindawi.com
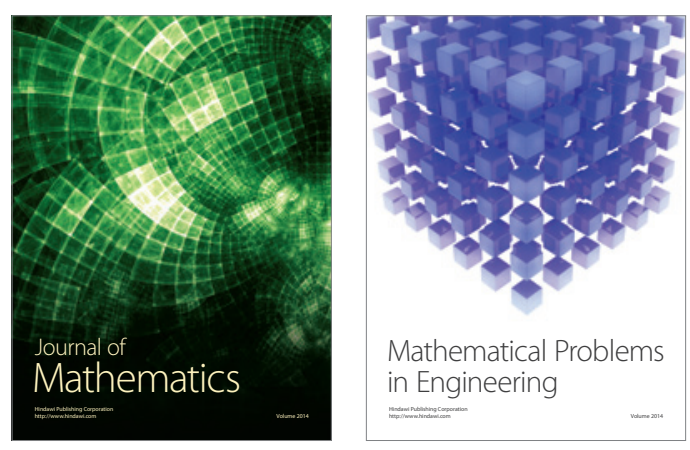

Mathematical Problems in Engineering
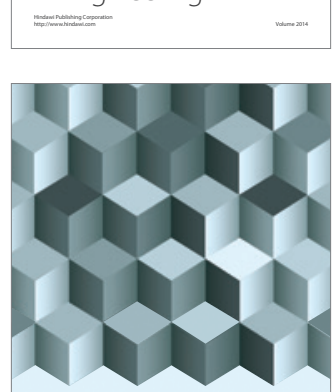

Journal of

Function Spaces
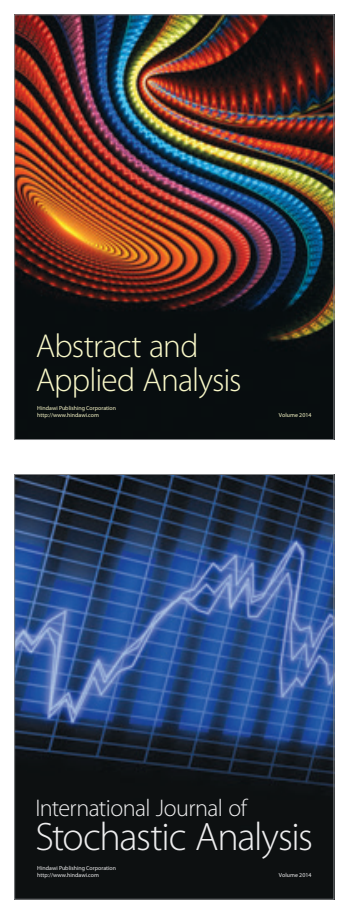

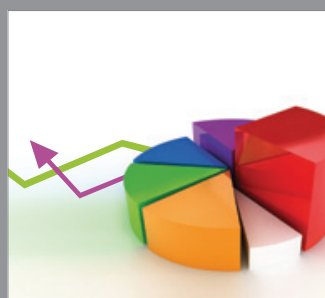

ournal of

Probability and Statistics

Promensencen
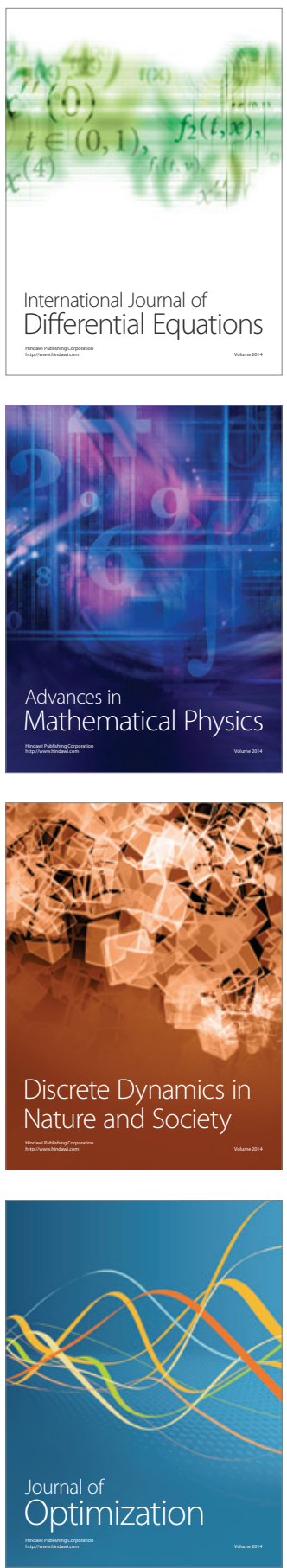